\title{
BMJ Open Protocol for a pilot randomised controlled trial of metformin in pre- diabetes after kidney transplantation: the Transplantation and Diabetes (Transdiab) study
}

\author{
Basil Alnasrallah, ${ }^{1}$ Helen Pilmore, ${ }^{1,2}$ Paul Manley ${ }^{1}$
}

To cite: Alnasrallah B, Pilmore H, Manley P. Protocol for a pilot randomised controlled trial of metformin in pre-diabetes after kidney transplantation: the Transplantation and Diabetes (Transdiab) study. BMJ Open 2017;7:e016813. doi:10.1136/ bmjopen-2017-016813

- Prepublication history and additional material are available. To view these files please visit the journal online (http://dx.doi. org/10.1136/bmjopen-2017016813).

Received 26 March 2017 Revised 17 June 2017 Accepted 23 June 2017

CrossMark

${ }^{1}$ Department of Nephrology, Auckland City Hospital, Auckland, New Zealand ${ }^{2}$ Department of Medicine, University of Auckland, Auckland, New Zealand

Correspondence to Dr Basil Alnasrallah; polbeas@yahoo.com

\section{ABSTRACT}

Introduction Post-transplant diabetes mellitus (PTDM) is a common complication of kidney transplantation and is associated with significant morbidity and mortality. In the general population, metformin has been used for diabetes prevention in high-risk individuals. Improving insulin sensitivity is one of many proven favourable effects of metformin. Despite the high incidence of PTDM in kidney transplant recipients, there is a lack of evidence for the role of metformin in the prevention of diabetes in this setting.

Methods and analysis Transplantation and Diabetes (Transdiab) is a single-centre, unblinded, pilot randomised controlled trial assessing the feasibility, tolerability and efficacy of metformin after renal transplantation in patients with impaired glucose tolerance (IGT). Participants will undergo an oral glucose tolerance test in the 4-12 weeks post-transplantation; those with IGT will be randomised to standard care or standard care and metformin $500 \mathrm{mg}$ twice daily, and followed up for 12 months. The primary outcomes of the study will be the feasibility of recruitment, the tolerability of metformin assessed using the Gastrointestinal Symptom Rating Scale at 3 and 12 months, and the efficacy of metformin assessed by morning glucose and glycated haemoglobin at 3, 6, 9 and 12 months.

Ethics and dissemination Despite the significant morbidity and mortality of PTDM, there are currently no randomised clinical trials assessing pharmacological interventions for its prevention after kidney transplantation. The Transdiab trial will thus provide important data on the feasibility, safety, tolerability and efficacy of metformin after renal transplantation in patients with IGT; this will facilitate undertaking larger multicentre trials of interventions to reduce the incidence or severity of diabetes after kidney transplantation. This study has been approved by the Northern B Health and Disability Ethics Committee of the Ministry of Health in New Zealand. On study completion, results are expected to be published in a peer-reviewed journal.

Trial registration number Australian New Zealand Clinical Trials Registry Number: ACTRN12614001171606.
Strengths and limitations of this study

- This study has the potential to address more than one important cause of morbidity and mortality in kidney transplant patients with one simple approach.

- It is difficult to provide sufficient data for clinical effectiveness of the measure used.

- Safety and tolerability outcomes are likely to be informative and crucial for larger future randomised controlled trials.

\section{INTRODUCTION}

End-stage kidney disease (ESKD) is a major public health problem with increasing prevalence worldwide. In 2013, there were 4156 people receiving renal replacement therapy for ESKD in New Zealand. This represents a population rate of 931 people per million. Of these, $1572 \quad(38 \%)$ had a functioning kidney transplant. ${ }^{1} \quad$ Kidney transplantation represents the optimal form of renal replacement therapy and is associated with significantly improved survival, ${ }^{2}{ }^{3}$ quality of life ${ }^{4}$ and reduced costs over time ${ }^{5}$ compared with dialysis treatment. However, transplant recipients still have increased mortality compared with the general population. ${ }^{6}$ This is predominantly driven by increased cardiovascular disease, as cardiovascular-related deaths post-transplant constitute over $50 \%$ of all deaths. ${ }^{78}$ An important risk factor for this marked risk is post-transplant diabetes mellitus (PTDM), previously known as new onset diabetes after transplantation.

PTDM is a common complication after transplantation, with a reported incidence as high as $50 \%{ }^{9}$ and significant impact on morbidity and mortality in transplant recipients. Patients with PTDM have significantly higher rates of cardiovascular disease, cardiovascular death and overall mortality 
with a doubling in all-cause mortality and a tripling in cardiovascular events. ${ }^{10-12}$ The mortality risk extends to include other forms of impaired glucose metabolism after transplantation as patients with impaired glucose tolerance (IGT) have an increased long-term mortality risk comparable with those with PTDM. ${ }^{13}$ In addition, PTDM is also associated with increased death censored graft failure. ${ }^{12} 14$

In 2003, an expert committee set forth the International Consensus Guidelines for the diagnosis and management of PTDM. ${ }^{15}$ This was further revised in 2013 with The International Expert Panel setting forth the recommendations for PTDM and future directions of research. ${ }^{16}$ The research recommendations highlighted the need for trials to help delay or prevent PTDM. The guidelines for PTDM are largely based on those of the WHO for the diagnosis of pre-diabetic states (impaired fasting glucose (IFG) and IGT) and diabetes mellitus. ${ }^{17}$ Multiple risk factors have been associated with the development of PTDM; the use of diabetogenic immunosuppressive medications (such as corticosteroids, ${ }^{18}$ calcineurin inhibitors $^{1219}$ and mammalian target of rapamycin inhibitors ${ }^{20}$ ) are all associated with impaired glucose metabolism. In addition, the significant incidence of weight gain and obesity after transplantation plays a role in insulin resistance in these patients, with average weight gain in the first year after transplantation of $10 \%-35 \% .^{21-23}$ The use of immunosuppressive medications (such as corticosteroids), improvement in well-being and the relaxation of dietary restrictions after transplantation are possible causes for this weight gain. ${ }^{24-26}$

Another well-identified risk factor for the development of diabetes in both transplant recipients and the general population is the status of pre-diabetes (IGT or IFG), with IFG (fasting plasma glucose $\geq 6.1$ and $<7 \mathrm{mmol} / \mathrm{L}$ ) or IGT (fasting plasma glucose $<7 \mathrm{mmol} / \mathrm{L}$ and 2-hour plasma glucose after an oral glucose tolerance test (OGTT) $\geq 7.8$ and $<11.1 \mathrm{mmol} / \mathrm{L}$ ) defining hyperglycaemic conditions at risk of the future development of diabetes. ${ }^{12}$ In non-transplanted patients, some $70 \%$ of individuals with IFG and/or IGT can expect to go on to develop clinical type 2 diabetes at some time in the future. ${ }^{27}$ That risk is also significant for transplant patients with $15 \%$ of patients with IGT developing PTDM over 1 year $^{28}$ and $27 \%$ over 6 years. $^{29}$

Given the significant adverse clinical outcomes associated with diabetes, multiple measures have been introduced to prevent or delay the onset of diabetes in high-risk individuals. Current guidelines recommend lifestyle modifications in the management of pre-diabetes or diabetes, aiming to achieve weight loss in overweight or obese patients. $^{30} 31$

However, weight loss arising from intensive lifestyle interventions is difficult to maintain over the long term. ${ }^{32}$ Therefore, the current guidelines recommend the use of pharmacological antidiabetic therapy immediately after the diagnosis of diabetes for patients considered unlikely to benefit sufficiently from lifestyle intervention alone. ${ }^{33} 34$
Pharmacological therapy such as metformin, thiazolidinediones and alpha-glucosidase inhibitors have been shown to effectively delay or prevent the conversion of pre-diabetes to diabetes in non-transplant patients; however, metformin is the only pharmacological agent recommended for the prevention or delay of type 2 diabetes in at-risk subjects. This is mainly due to its effectiveness, good tolerability and low cost. 303134

No similar trials have been carried out in transplant recipients. These patients have a high risk of developing PTDM and the subgroup with IGT are likely to be at even greater risk. Therefore, this group might achieve a significant benefit from starting metformin in addition to lifestyle advice.

We will be conducting a pilot randomised controlled trial assessing the safety, tolerability and efficacy of metformin in renal transplant recipients with IGT. The trial will also provide data on the feasibility of recruiting eligible patients with IGT post-transplantation to be started on metformin, which will help to plan conducting larger trials aiming to reduce the incidence or severity of diabetes after kidney transplantation.

\section{METHODS AND ANALYSIS \\ Study design}

Transplantation and Diabetes (Transdiab) is a singlecentre, unblinded, parallel-group, randomised controlled trial. An outline of the study is shown in figure 1 .

\section{Study aims and hypothesis}

The primary aim of the PTDM is to evaluate the feasibility, safety, tolerability and efficacy of metformin in kidney transplant patients with IGT. The secondary outcomes are those that will help evaluate the additional benefits of metformin on lipid profile, change in weight and cardiovascular events. This will be done in a superiority framework.

We hypothesise that recruiting non-diabetic patients after renal transplantation to have OGTTs in order to identify and pharmacologically manage those with IGT is feasible. We also hypothesise that using metformin within our inclusion criteria is safe with acceptable tolerability in the first year after renal transplantation. Furthermore, it is likely that metformin will have a favourable effect on glycated haemoglobin (HbAlc), morning glucose, lipid profile, excess weight gain and cardiovascular events.

\section{Target population and eligibility criteria}

The target population for the trial is adult kidney transplant recipients who receive kidney transplantation in our centre between 30 November 2014 and 30 November 2016.

\section{Inclusion criteria}

Inclusion criteria will include the following: age $>18$ years, non-diabetic kidney transplant recipient, willing and able to participate in all trial investigations for the duration of 


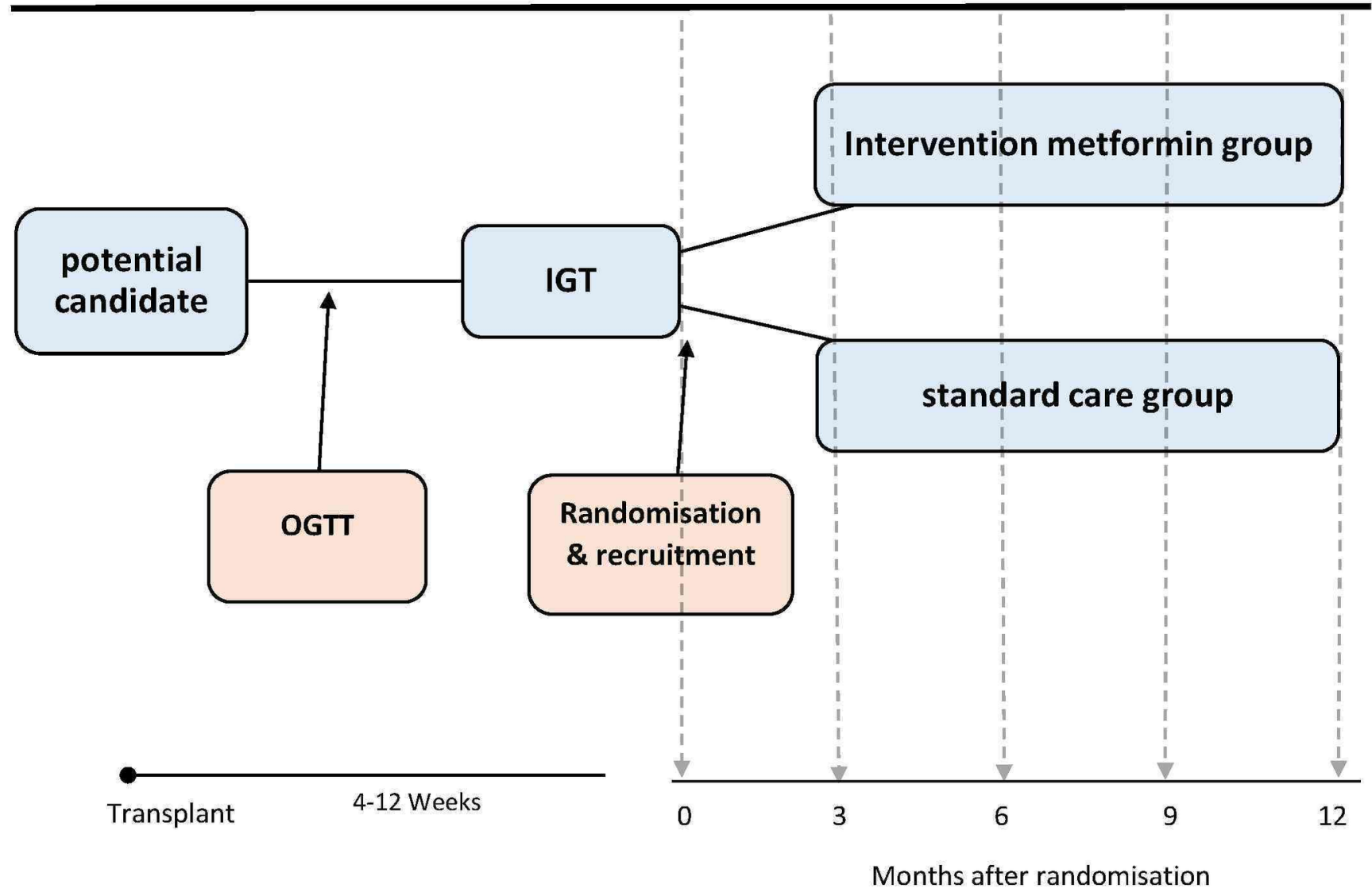

Figure 1 Flow chart of Transdiab trial. IGT, impaired glucose tolerance; OGTT, oral glucose tolerance test.

trial follow-up and ability to provide a written informed consent.

\section{Exclusion criteria}

Exclusion criteria will include the following: known diabetes mellitus at the time of transplant (whether on antidiabetic medications or not), history of antidiabetic therapy (oral or insulin), steroid pulse therapy (intravenous or oral) in the 2 weeks prior to OGTT, estimated glomerular filtration rate (eGFR) by the Modification of Diet in Renal Disease formula $\leq 30 \mathrm{~mL} / \mathrm{min} / 1.73 \mathrm{~m}^{2} \mathrm{BSA}$ (body surface area), unable to consent, pregnancy or breast feeding, current alcohol or other substance abuse and any major illness or comorbidity that may result in death in 12 months as assessed by the treating physician.

\section{Recruitment and randomisation}

Potential participants will be identified during their initial inpatient stay following transplantation at Auckland City Hospital or through the renal transplant outpatient clinic following discharge. Eligible transplant recipients will be invited to participate, given detailed information about the trial and, if agreeable, will be asked to provide a written informed consent by one of the investigators. In order to participate, transplant recipients will need to provide informed consent and have the OGTT within 4-12 weeks after the transplant procedure. The consent form and patient information sheet are available in the online supplementary file 1. Consented patients will have their information collected and stored securely by the research fellow, physically in the research office and electronically on a password-protected computer. The research fellow will also generate the allocation sequence, enrol participants and assign the groups.

Participants who have a 2-hour postload glucose between 7.8 and $11.1 \mathrm{mmol} / \mathrm{L}$ on OGTT (75 g anhydrous glucose dissolved in water) will be randomised. Randomisation will be performed using a computer-generated sequence allocation in blocks of four in a 1:1 ratio to receive either standard care or standard care and metformin $500 \mathrm{mg}$ twice daily.

\section{Standard care group}

Participants randomised to the standard care group will receive standard post-transplant care as per usual local practice. This includes immunosuppressive medications to prevent rejection and other treatments under the direction of the treating physician and renal transplant team. Standard immunosuppression includes a calcineurin inhibitor (ciclosporin or tacrolimus as determined by the 
treating physician), mycophenolate, steroids and basiliximab as induction therapy.

The current lifestyle standard care after kidney transplantation at Auckland City Hospital includes advice for regular exercise three times weekly and a nutritional assessment by a renal dietician during the inpatient stay. The nutrition care involves giving patients resources guiding them on healthy eating and food safety after transplant.

\section{Intervention group}

Patients randomised to the intervention group will, in addition to standard care, receive metformin $500 \mathrm{mg}$ twice daily. This will commence at randomisation and continue for 12 months. The decision to continue with metformin beyond 12 months of follow-up is left to the discretion of the caring physician.

\section{Primary outcomes}

\section{Feasibility of recruitment}

Feasibility of recruitment will be assessed by the ratio of the number of randomised patients to the number of patients screened with OGTTs.

\section{Tolerability of metformin}

Tolerability of metformin will be assessed using the Gastrointestinal Symptom Rating Scale (GSRS), a tool that has been validated to assess symptoms in gastrointestinal disorders such as gastro-oesophageal reflux disease and irritable bowel syndrome ${ }^{3536}$ at baseline, 3 and 12 months postrandomisation.

\section{Efficacy of metformin}

Efficacy of metformin will be assessed by HbAlc and morning glucose levels at baseline, 3, 6, 9 and 12 months post-randomisation.

\section{Secondary outcome}

Secondary outcomes will include the following: change in body weight; all adverse events; lipid profile at 3, 6 , 9 and 12 months; major cardiac events; and proportion of patients who revert to normal glucose metabolism on OGTT at 12 months. When clinically indicated, lactate levels will be checked. Also, discontinuation of metformin or reduction of dose due to adverse effects will be recorded.

\section{Monitoring}

Interim results will be reviewed and analysed by the lead investigator every 3 months, and the decision for early termination of the trial will be considered if serious concerns about patients' safety are encountered. Full annual analysis of results will be carried out until the completion of the trial. If important changes to the protocol are to take place, they will be conveyed to the sponsor on the annual progress report and also to the affected patients and caring physicians. The access of the final trial dataset will be available to the primary investigator.

\section{Statistical analysis}

The first primary outcome, feasibility of recruitment, will be reported as a ratio of the number of randomised patients to the number of patients screened with OGTT at the conclusion of the study.

The second primary outcome, tolerability of metformin, will be reported as GSRS at 3 and 12 months and compared between the two groups using an analysis of covariance (ANCOVA) adjusted for GSRS score at baseline. The third primary outcome, efficacy of metformin, will be reported by measuring morning glucose and HbAlc 3, 6, 9 and 12 months postrandomisation and compared between the two groups using an ANCOVA adjusted for morning glucose and HbAlc at baseline.

Linear regression will be used to identify significant predictors other than group allocation associated with changes in efficacy, tolerability and metabolic parameters over time. Variables identified as significant will be analysed with pairwise comparisons. Comparisons between groups for differences in categorical variables will be conducted using $\chi^{2}$ test or Fisher's exact test. All statistical analyses will be performed using appropriate statistical software, such as STATA or SPSS (version 24.0). The level of statistical significance will be set at probability level of $<0.05$.

\section{Ethical considerations}

Ethical approval has been obtained through the Northern B Health and Disability Ethics Committee of the Ministry of Health in New Zealand. Ethics approval number is 14/ $\mathrm{STH} / 129$.

\section{DISCUSSION}

This trial will provide evidence on the feasibility, safety, tolerability and efficacy of metformin in patients with IGT after renal transplantation. In addition, the study will also examine in detail changes in other metabolic indices, including body mass index, weight change, blood pressure, lipid profile and cardiac events.

The extensive local and international experience with metformin and the significant potential benefits are likely to encourage patients and physicians to take active part in the study. Based on evidence from the literature, $43 \%$ of stable kidney transplant patients ( $>6$ months after transplant) will have new impaired glucose metabolism after transplantation with new IGT and/or IFG affecting $32 \%$ of all recipients. ${ }^{37}$ Therefore, we hypothesise that performing OGTTs in non-diabetic patients after renal transplantation and subsequently starting those with IGT on metformin will prove feasible.

Although generally safe, gastrointestinal side effects are not uncommon with the use of metformin; these are generally well tolerated and infrequently cause treatment discontinuation. ${ }^{38}$ The long-term experience with metformin in the diabetic general population has established good tolerability and an acceptable safety profile ${ }^{39}$ also in addition to good adherence by patients. ${ }^{40} \mathrm{~A}$ major 
and probably overstated potential side effect is lactic acidosis. Historically, a very low risk of lactic acidosis linked to the use of metformin has been reported. ${ }^{41}$ More recent high-quality evidence has shown no risk related to metformin in the general population with type 2 diabetes. ${ }^{42}$ The risk is probably higher in patients with reduced glomerular filtration rate (GFR) as metformin is more likely to accumulate, but a systematic review found no cases of lactic acidosis despite almost half of the studies involved allowing inclusion of patients with a serum creatinine above $1.5 \mathrm{mg} / \mathrm{dL}(133 \mu \mathrm{mol} / \mathrm{L}){ }^{43}$ Given the relative safety of metformin and significant benefit, the expansion of its use has been recommended to include patients with mild to moderate kidney disease (eGFRs, $30-60 \mathrm{~mL} / \mathrm{min}$ per $\left.1.73 \mathrm{~m}^{2}\right) .{ }^{44}$

Although physicians might have reservations starting metformin in renal transplant recipients as they commonly have abnormal GFRs with frequent fluctuations, the recent evidence provides further reassurance in the safety and tolerability of metformin even at moderate renal impairment. Therefore, we hypothesise that the use of metformin in these patients is safe and well tolerated.

Metformin has other favourable effects besides improving insulin sensitivity, some of which can be of substantial benefit to transplant patients. Most notable are the effects on cardiovascular disease ${ }^{45}$ and weight gain, ${ }^{46}$ with current ongoing research assessing these benefits even in pre-diabetic patients. ${ }^{47}$

Furthermore, metformin has been shown to reduce the incidence of cancersand cancer-related mortality. ${ }^{449}$ Renal transplant recipients have high cancer-related morbidity and mortality with cancer risk almost three times higher than the general population. ${ }^{50} 51$

The outcomes from this research will provide important data on the feasibility of conducting larger multicentre randomised trials of metformin in patients with IGT to reduce the incidence and severity of diabetes in kidney transplant recipients. This study will also provide transplant physicians with more confidence in using metformin in renal transplant recipients if no significant adverse events are encountered. There is a potential risk of bias as the participants are not blinded to the treatment; this could be addressed with the use of placebo in a larger trial.

Measurement of efficacy of the use of metformin is likely to require a large multicentre trial to have a sufficient number of patients for a significant difference. ${ }^{40}$ We anticipate that by determining feasibility and safety in this pilot study, we will pave the way for future larger randomised controlled trials. We intend to communicate our findings via publications and presentations in national and international meetings.

\section{CONCLUSIONS}

The Transdiab study will provide important data on the feasibility, safety and efficacy of metformin in patients with IGT after renal transplantation. With PTDM being an important cause of morbidity and mortality in renal transplant patients, this study will address a significant gap in current evidence regarding optimal care after kidney transplantation.

Contributors The three authors made substantial contributions to the design of the work, obtaining ethics approval, registering the trial and final write-up of the paper. PM: main contributor to the initial design of the research and subsequently acquiring ethics approval, registering the study and submitting the proposal for the funding grant. Substantial contribution to the work draft. Reviewing and amending the final version for publishing. HP: lead investigator. Contributed to the initial conception of the work and revised the initial design critically for ingenuity. Provided further insight into the potential limitations and provided the required support for obtaining the funding grant. Supervised the designated fellow in following the study protocol and conducting the appropriate analysis. Reviewed and amended the final version for publishing. Steering investigator, conveys annual progress to the funder and review progress with the research fellow. Will have the access to the final database and any related agreements. BA: substantial contribution to the interpretation of data for the work. Also, drafted the final protocol after reviewing the relevant literature and submitted it for the other authors' comments and amended it accordingly. Identified the limitations in the study and the practical challenges while carrying out the study and initial analysis. Generates the allocation sequence, enrols participants and assigns participants to interventions. Also, responsible for the entry and storage of the study's data in the research computer. All authors agreed to be accountable for all aspects of the work in ensuring that any issues related to any part of the work are appropriately investigated and resolved.

Funding The study received a grant from A+ charitable trust in Auckland, New Zealand for the research fellow salary and stationary costs (grantnumber 6218).

Disclaimer The funding source had no role in the design of this study and will not have any role during its execution, analyses, interpretation of the data or decision to submit results.

Competing interests None declared.

Patient consent Obtained.

Ethics approval Health and Disability Ethics Committees-New Zealand.

Provenance and peer review Not commissioned; externally peer reviewed.

Open Access This is an Open Access article distributed in accordance with the Creative Commons Attribution Non Commercial (CC BY-NC 4.0) license, which permits others to distribute, remix, adapt, build upon this work non-commercially, and license their derivative works on different terms, provided the original work is properly cited and the use is non-commercial. See: http://creativecommons.org/ licenses/by-nc/4.0/

(C) Article author(s) (or their employer(s) unless otherwise stated in the text of the article) 2017. All rights reserved. No commercial use is permitted unless otherwise expressly granted.

\section{REFERENCES}

1. ANZDATA Registry. 37th Report, Preliminary Report 2014: Summary of Dialysis and Transplantation in Australia and New Zealand. Australia and New Zealand Dialysis and Transplant Registry, Adelaide, Australia, 2015. http://www.anzdata.org.au.

2. Port FK, Wolfe RA, Mauger EA, et al. Comparison of survival probabilities for dialysis patients vs cadaveric renal transplant recipients. JAMA 1993;270:1339-43.

3. McDonald SP, Russ GR. Survival of recipients of cadaveric kidney transplants compared with those receiving dialysis treatment in Australia and New Zealand, 1991-2001. Nephrol Dial Transplant 2002;17:2212-9.

4. Laupacis A, Keown P, Pus N, et al. A study of the quality of life and cost-utility of renal transplantation. Kidney Int 1996;50:235-42.

5. Howard K, Salkeld G, White S, et al. The cost-effectiveness of increasing kidney transplantation and home-based dialysis. Nephrology 2009;14:123-32.

6. van Walraven C, Manuel DG, Knoll G. Survival trends in ESRD patients compared with the general population in the United States. Am J Kidney Dis 2014;63:491-9.

7. Hjelmesaeth J, Hartmann A, Leivestad T, et al. The impact of earlydiagnosed new-onset post-transplantation diabetes mellitus on survival and major cardiac events. Kidney Int 2006;69:588-95. 
8. Cosio FG, Pesavento TE, Kim S, et al. Patient survival after renal transplantation: IV. Impact of post-transplant diabetes. Kidney Int 2002;62:1440-6.

9. Balla A, Chobanian M. New-onset diabetes after transplantation: a review of recent literature. Curr Opin Organ Transplant 2009;14:375-9.

10. Revanur VK, Jardine AG, Kingsmore DB, et al. Influence of diabetes mellitus on patient and graft survival in recipients of kidney transplantation. Clin Transplant 2001;15:89-94.

11. Hjelmesaeth J, Hartmann A, Leivestad T, et al. The impact of earlydiagnosed new-onset post-transplantation diabetes mellitus on survival and major cardiac events. Kidney Int 2006;69:588-95.

12. Kasiske BL, Snyder JJ, Gilbertson D, et al. Diabetes mellitus after kidney transplantation in the United States. Am J Transplant 2003;3:178-85

13. American Diabetes Association. Standards of medical care in diabetes-2014. Diabetes Care 2015;38(Suppl 1):S31-3.

14. Cole $\mathrm{EH}$, Johnston $\mathrm{O}$, Rose $\mathrm{CL}$, et al. Impact of acute rejection and new-onset diabetes on long-term transplant graft and patient survival. Clin J Am Soc Nephrol 2008;3:814-21.

15. New-onset diabetes after transplantation: 2003 International consensus guidelines. Proceedings of an international expert panel meeting. Barcelona, Spain, 2003

16. Sharif A, Hecking M, de Vries AP, et al. Proceedings from an international consensus meeting on posttransplantation diabetes mellitus: recommendations and future directions. Am J Transplant 2014;14:1992-2000

17. Alberti KG, Zimmet PZ. Definition, diagnosis and classification of diabetes mellitus and its complications. Part 1: diagnosis and classification of diabetes mellitus provisional report of a WHO consultation. Diabet Med 1998;:15:539-53.

18. Rodrigo E, Fernández-Fresnedo G, Valero R, et al. New-onset diabetes after kidney transplantation: risk factors. J Am Soc Nephrol 2006;17:S291-S295.

19. Woodward RS, Schnitzler MA, Baty J, et al. Incidence and cost of new onset diabetes mellitus among U.S. wait-listed and transplanted renal allograft recipients. Am J Transplant 2003;3:590-8.

20. Johnston O, Rose CL, Webster AC, et al. Sirolimus is associated with new-onset diabetes in kidney transplant recipients. J Am Soc Nephrol 2008;19:1411-8.

21. Jaggers HJ, Allman MA, Chan M. Changes in clinical profile and dietary considerations after renal transplantation. J Renal Nutr 1996;6:12-20.

22. Johnson CP, Gallagher-Lepak S, Zhu YR, et al. Factors influencing weight gain after renal transplantation. Transplantation 1993;56:822-6.

23. Teplan V, Poledne R, Schück O, et al. Hyperlipidemia and obesity after renal transplantation. Ann Transplant 2001;6:21-3.

24. Baum CL, Thielke K, Westin E, et al. Predictors of weight gain and cardiovascular risk in a cohort of racially diverse kidney transplant recipients. Nutrition 2002;18:139-46.

25. Heaf J, Jakobsen U, Tvedegaard E, et al. Dietary habits and nutritional status of renal transplant patients. J Ren Nutr 2004;14:20-5.

26. Ryan KJ, Casas JM, Mash LE, et al. The effect of intensive nutrition interventions on weight gain after kidney transplantation: protocol of a randomised controlled trial. BMC Nephrol 2014;15:148.

27. Nathan DM, Davidson MB, DeFronzo RA, et al. Impaired fasting glucose and impaired glucose tolerance: implications for care. Diabetes Care 2007;30:753-9.

28. Hornum M, Jørgensen KA, Hansen JM, et al. New-onset diabetes mellitus after kidney transplantation in Denmark. Clin J Am Soc Nephrol 2010;5:709-16.

29. Hagen M, Hjelmesaeth J, Jenssen T, et al. A 6-year prospective study on new onset diabetes mellitus, insulin release and insulin sensitivity in renal transplant recipients. Nephrol Dial Transplant 2003;18:2154-9.

30. American Diabetes Association. Standards of medical care in diabetes-2014. Diabetes Care 2015;38(Suppl 1):S31-3.

31. Paulweber B, Valensi P, Lindström J, et al. A European evidencebased guideline for the prevention of type 2 diabetes. Horm Metab Res 2010;42 Suppl 1(Suppl1):S3-36.

32. Knowler WC, Fowler SE, Hamman RF, et al. 10-year follow-up of diabetes incidence and weight loss in the Diabetes Prevention Program Outcomes Study. Lancet 2009;374:1677-86.

33. Inzucchi SE, Bergenstal RM, Buse JB, et al. Management of hyperglycemia in type 2 diabetes: a patient-centered approach. Position statement of the American Diabetes Association (ADA) and the European Association for the Study of Diabetes (EASD). Diabetologia 2012:55:1577-96.

34. Hostalek U, Gwilt M, Hildemann S. Therapeutic use of metformin in prediabetes and diabetes prevention. Drugs 2015;75:1071-94.

35. Kulich KR, Madisch A, Pacini F, et al. Reliability and validity of the Gastrointestinal Symptom Rating Scale (GSRS) and Quality of Life in Reflux and Dyspepsia (QOLRAD) questionnaire in dyspepsia: a sixcountry study. Health Qual Life Outcomes 2008;6:12.

36. Svedlund J, Sjödin I, Dotevall G. GSRS - a clinical rating scale for gastrointestinal symptoms in patients with irritable bowel syndrome and peptic ulcer disease. Dig Dis Sci 1988;33:129-34.

37. Hecking M, Kainz A, Werzowa J, et al. Glucose metabolism after renal transplantation. Diabetes Care 2013;36:2763-71.

38. Scarpello JH. Review: optimal dosing strategies for maximising the clinical response to metformin in type 2 diabetes. $\mathrm{Br} J$ Diabetes Vasc Dis 2001;1:28-36.

39. Bailey CJ, Campbell IW, Chan JCN, et al, eds. Metformin, the gold standard: a scientific handbook. Chichester: Wiley, 2007:173-92.

40. Knowler WC, Barrett-Connor E, Fowler SE, et al. Reduction in the incidence of type 2 diabetes with lifestyle intervention or metformin. N Engl J Med 2002;346:393-403.

41. Stang M, Wysowski DK, Butler-Jones D. Incidence of lactic acidosis in metformin users. Diabetes Care 1999;22:925-7.

42. Cryer DR, Nicholas SP, Henry DH, et al. Comparative outcomes study of metformin intervention versus conventional approach the COSMIC Approach Study. Diabetes Care 2005;28:539-43.

43. Salpeter SR, Greyber E, Pasternak GA, et al. Risk of fatal and nonfatal lactic acidosis with metformin use in type 2 diabetes mellitus. Cochrane Database Syst Rev 2010:CD002967.

44. Inzucchi SE, Lipska KJ, Mayo H, et al. Metformin in patients with type 2 diabetes and kidney disease: a systematic review. JAMA 2014;312:2668-75.

45. UK Prospective Diabetes Study (UKPDS) Group. Effect of intensive blood-glucose control with metformin on complications in overweight patients with type 2 diabetes (UKPDS 34). Lancet 1998;352:854-65.

46. Seifarth C, Schehler B, Schneider HJ. Effectiveness of metformin on weight loss in non-diabetic individuals with obesity. Exp Clin Endocrinol Diabetes 2013;121:27-31.

47. University of Oxford Diabetes Trials Unit. Glucose lowering in nondiabetic hyperglycaemia trial (ongoing). https://www.dtu.ox.ac.uk/ GLINT/ (accessed Jan 2015).

48. Libby G, Donnelly LA, Donnan PT, et al. New users of metformin are at low risk of incident cancer: a cohort study among people with type 2 diabetes. Diabetes Care 2009;32:1620-5.

49. Landman GW, Kleefstra N, van Hateren KJ, et al. Metformin associated with lower cancer mortality in type 2 diabetes: ZODIAC-16. Diabetes Care 2010;33:322-6.

50. Kasiske BL, Snyder JJ, Gilbertson DT, et al. Cancer after kidney transplantation in the United States. Am J Transplant 2004;4:905-13.

51. Vajdic CM, McDonald SP, McCredie MR, et al. Cancer incidence before and after kidney transplantation. JAMA 2006;296:2823. 